\title{
MESSENGER and Venus Express observations of the solar wind interaction with Venus
}

\author{
James A. Slavin, ${ }^{1}$ Mario H. Acuña, ${ }^{2,3}$ Brian J. Anderson, ${ }^{4}$ Stas Barabash,${ }^{5}$ Mehdi Benna, ${ }^{2}$ \\ Scott A. Boardsen, ${ }^{1}$ Markus Fraenz, ${ }^{6}$ George Gloeckler, ${ }^{7}$ Robert E. Gold, ${ }^{4}$ George C. Ho, ${ }^{4}$ \\ Haje Korth, ${ }^{4}$ Stamatios M. Krimigis, ${ }^{4}$ Ralph L. McNutt Jr., ${ }^{4}$ Jim M. Raines, ${ }^{7}$
} Menelaos Sarantos, ${ }^{1}$ Sean C. Solomon, ${ }^{8}$ Tielong Zhang, ${ }^{9}$ and Thomas H. Zurbuchen ${ }^{7}$

Received 26 February 2009; revised 30 March 2009; accepted 6 April 2009; published 12 May 2009.

[1] At 23:08 UTC on 5 June 2007 the MESSENGER spacecraft reached its closest approach altitude of $338 \mathrm{~km}$ during its final flyby of Venus en route to its 2011 orbit insertion at Mercury. The availability of the simultaneous Venus Express solar wind and interplanetary magnetic field measurements provides a rare opportunity to examine the influence of upstream conditions on this planet's solar wind interaction. We present MESSENGER observations of new features of the Venus - solar wind interaction including hot flow anomalies upstream of the bow shock, a flux rope in the near-tail and a two-point determination of the timescale for magnetic flux transport through this induced magnetosphere. Citation: Slavin, J. A., et al. (2009), MESSENGER and Venus Express observations of the solar wind interaction with Venus, Geophys. Res. Lett., 36, L09106, doi:10.1029/2009GL037876.

\section{Introduction}

[2] The solar wind interaction with Venus has been the subject of intensive investigation by the Venera 9 and 10 , Pioneer Venus Orbiter, and, most recently, Venus Express missions [Barabash et al., 2007; Zhang et al., 2008]. Venus has no measurable intrinsic magnetic field to shield it from the solar wind, which interacts directly with the planet's ionosphere and upper atmosphere. The resulting solar wind interaction is remarkably complex, with interplanetary conditions and solar extreme ultraviolet irradiance controlling the production and transport of ionospheric plasma, the degree of penetration of the interplanetary magnetic field (IMF) into the ionosphere, and the rate of volatile loss to

\footnotetext{
${ }^{1}$ Heliophysics Science Division, NASA Goddard Space Flight Center, Greenbelt, Maryland, USA.

${ }^{2}$ Solar System Exploration Division, NASA Goddard Space Flight Center, Greenbelt, Maryland, USA.

${ }^{3}$ Deceased 5 March 2009.

${ }^{4}$ Johns Hopkins University Applied Physics Laboratory, Laurel, Maryland, USA.

${ }^{5}$ Swedish Institute of Space Physics, Kiruna, Sweden.

${ }^{6}$ Max Planck Institute for Solar System Research, Katlenburg-Lindau, Germany.

${ }^{7}$ Department of Atmospheric, Oceanic and Space Sciences, University of Michigan, Ann Arbor, Michigan, USA.

${ }^{8}$ Department of Terrestrial Magnetism, Carnegie Institution of Washington, Washington, D. C., USA.

${ }^{9}$ Space Research Institute, Austrian Academy of Sciences, Graz, Austria.
}

Copyright 2009 by the American Geophysical Union. 0094-8276/09/2009GL037876 solar wind scavenging [Pérez-de-Tejada, 2004; McKennaLawlor et al., 2008].

[3] The close flyby of Venus by the MErcury Surface, Space ENvironment, GEochemistry, and Ranging (MESSENGER) spacecraft on 5-6 June 2007 offers a special opportunity to study the Venus plasma environment with Venus Express acting as a solar wind monitor. These rare two-point measurements provide the first observations of hot flow anomalies in the Venus bow shock and a large magnetic flux rope embedded in the cross-tail current sheet, as well as a new determination of the timescale for magnetic flux transport through the solar wind interaction region.

\section{MESSENGER and Venus Express Observations}

[4] The locations of the MESSENGER and Venus Express (VEX) spacecraft during 5-6 June 2007 are shown in Figure 1 using Venus Solar Orbital (VSO) coordinates. In this system $\mathrm{X}_{\mathrm{VSO}}$ is directed from the center of the planet toward the Sun, $Z_{\mathrm{VSO}}$ is normal to the Venus orbital plane and positive toward the north celestial pole, and $\mathrm{Y}_{\mathrm{VSO}}$ is positive in the direction opposite to orbital motion. Average Venus bow shock [Slavin et al., 1984] and ion composition boundary [Martinecz et al., 2008] models that span the MESSENGER trajectory are included for context. The MESSENGER spacecraft remained between $Z=-2 R_{V}$ and $+2 R_{V}$ during the encounter, where $R_{V}$ is the radius of Venus, while VEX was below the plane of the Venus orbit returning from apoapsis between $Z=-12 R_{V}$ and $-8 R_{V}$.

[5] The MESSENGER (black trace) and VEX (red trace) measurements of magnetic field $B$ are plotted together in Figure 2. The agreement between the two magnetic field data sets is within $\pm 1 \mathrm{nT}$ everywhere except, obviously, for the period when MESSENGER is in the Venus interaction region itself. The bottom plot of Figure 2a displays the IMF "clock angle," the angle that the projection of the IMF vector onto the $\mathrm{Y}-\mathrm{Z}$ plane makes relative to the $+\mathrm{Y}$ direction measured clockwise as viewed from the $+X$ direction. This angle is important because it defines the symmetry plane for magnetic field draping, and it rotated to different orientations throughout the MESSENGER encounter. VEX also measured the solar wind during the flyby (not shown) and determined that the solar wind speed, dynamic and static pressures, and Alfvén and sonic Mach numbers were all nearly constant and close to their mean values near Venus, $0.72 \mathrm{AU}$ from the Sun, of $420 \mathrm{~km} / \mathrm{s}, 5 \mathrm{nPa}$ and $0.02 \mathrm{nPa}$, and 7 and 8 , respectively. 

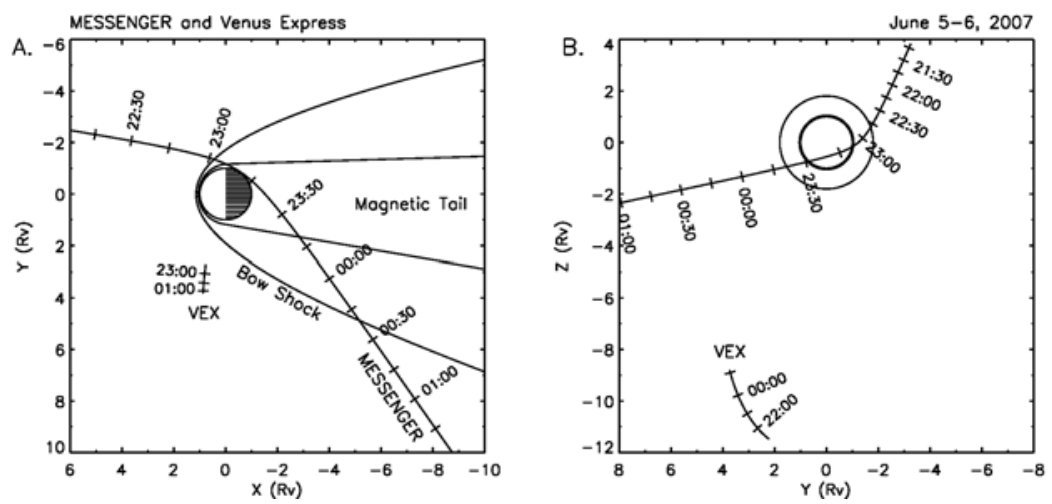

Figure 1. MESSENGER and Venus Express (VEX) trajectories projected into the VSO (a) X-Y and (b) Y-Z planes. Bow shock and ion composition boundaries from Slavin et al. [1984] and Martinecz et al. [2008] are displayed with $4^{\circ}$ of aberration.

[6] MESSENGER approached from the upstream direction, crossed the dawn-side foreshock, and encountered the bow shock at 22:57:54 UTC as shown in Figure 2b. After the dawn magnetosheath crossing the total field increased. The transition to these strong, low-variance magnetic field levels marks the crossing of the "magnetic pile-up boundary" (MPB) [Bertucci et al., 2003] and the entry into what is called the "magnetic barrier" or the "induced magnetosphere" [Zhang et al., 1991]. These current layers are essentially coincident with the "ion composition boundary" identified in plasma measurements [Martinecz et al., 2008] and explained in terms of viscous interactions [Pérezde-Tejada, 2004; McKenna-Lawlor et al., 2008]. The clockangle variations in the upstream IMF placed MESSENGER in the cross-tail current sheet as it moved around the dawn terminator and entered the near tail. The magnetic tail of
Venus consists of two lobes of strong, oppositely directed, relatively steady magnetic flux that maps back to the magnetic barrier [Saunders and Russell, 1986]. The two lobes are separated by a plasma sheet with a high ratio, $\beta>1$, of kinetic to magnetic pressure composed of solar wind and ionospheric plasma and planetary pick-up ions [Slavin et al., 1989; Barabash et al., 2007]. After multiple encounters with the cross-tail current layer, MESSENGER remained in the "toward" (i.e., $B_{X}>0$ ) lobe of the tail before exiting through a magnetopause-type current layer that forms the downstream extension of the MPB.

\section{Hot Flow Anomalies in the Venus Foreshock}

[7] Ahead of the inbound bow-shock crossing at 22:57:50, a well-developed foreshock was observed with
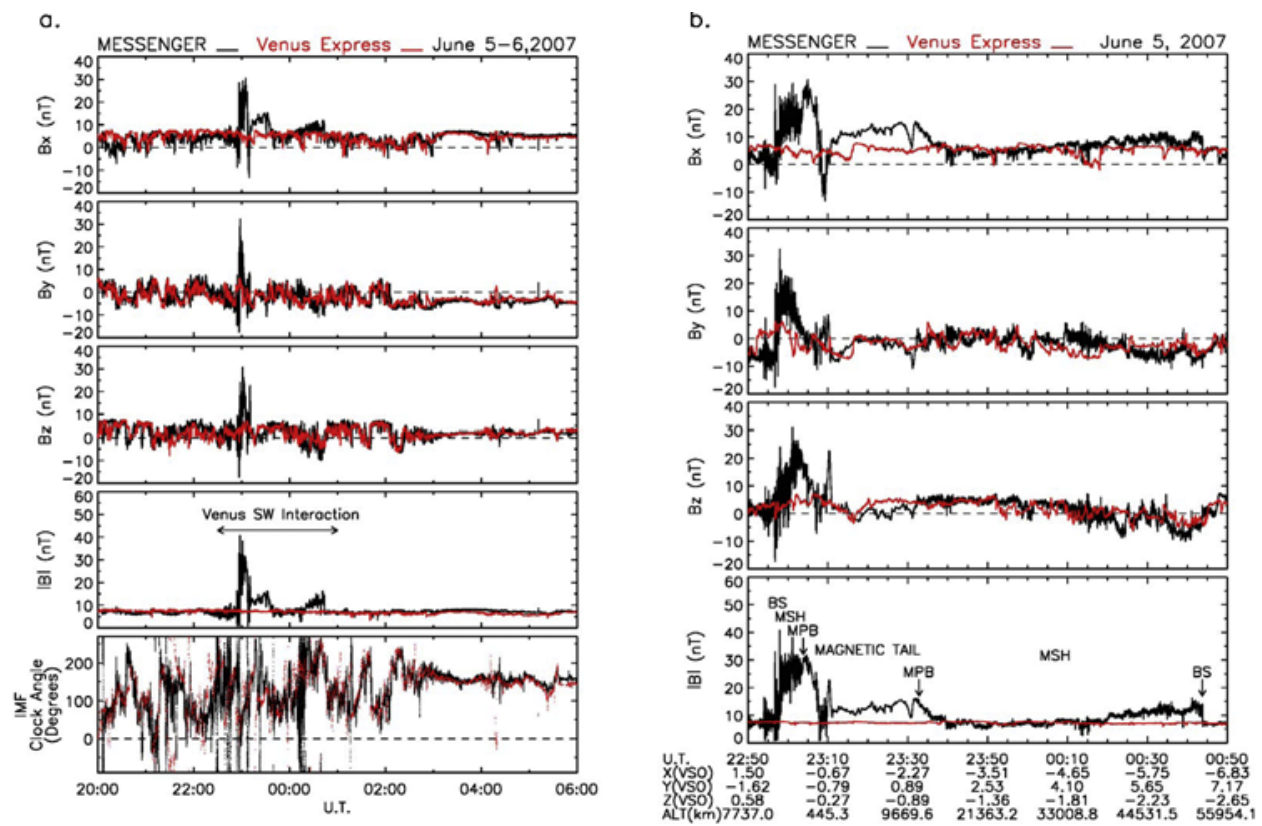

Figure 2. (a) MESSENGER and Venus Express magnetic field observations are displayed as black and red traces, respectively, in VSO coordinates from 20:00 UTC on 5 June 2007 to 06:00 UTC on 6 June 2007. (b) A closer view of these same magnetic field measurements from 22:50 to 00:50 UTC. BS = bow shock; $\mathrm{MSH}=$ magnetosheath; MPB = magnetic pile-up boundary. 

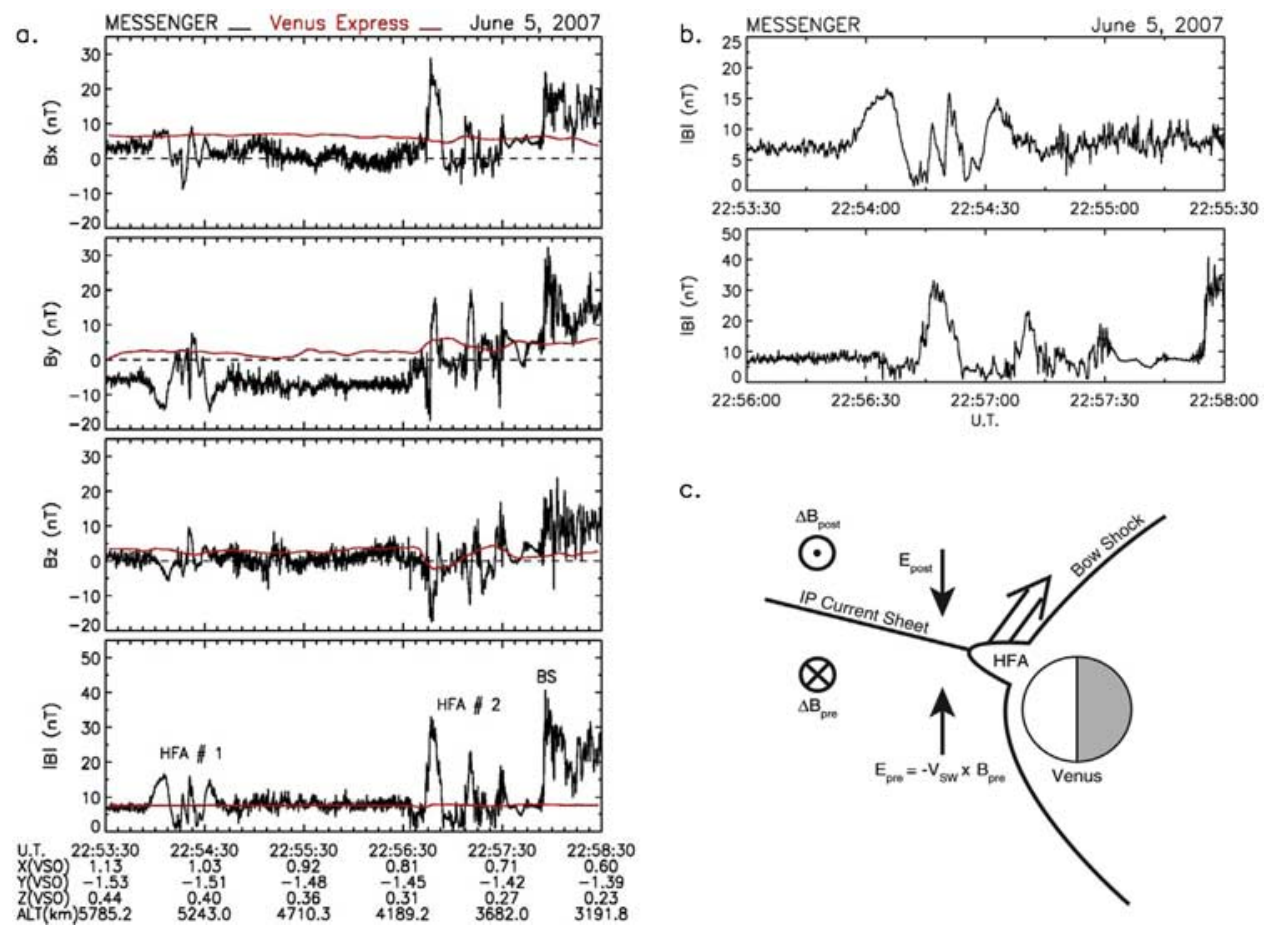

c.

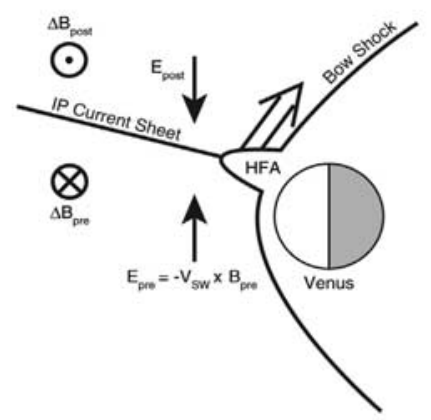

Figure 3. (a) Magnetic field measurements in the Venus foreshock just upstream of the bow shock showing the presence of two hot flow anomalies. (b) Detailed view of the two HFAs. (c) A schematic diagram, after Schwartz et al. [2000], showing the geometry and formation process for hot flow anomalies and the direction of the motional electric field observed by MESSENGER before, $E_{\text {pre, }}$ and after, $E_{\text {post }}$, the passage of the interplanetary (IP) current sheet.

extensive upstream waves, as shown in Figure 3a. Consistent with the wave activity, the angle between the local magnetic field and the normal to the bow shock determined from co-planarity, $\theta_{\mathrm{BN}}$, was generally less than $60^{\circ}$. Embedded within the foreshock are two groups, centered on 22:54:25 and 22:57:20, of strong, shock-like magnetic field increases that bound brief, $\sim 30-45 \mathrm{~s}$, intervals of decreases to near zero field intensity surrounding central peaks that are as strong as the outer enhancements. Observations at Earth have shown that these structures, termed "hot flow anomalies" (HFAs), are produced by the interaction of interplanetary current sheets with back-streaming ions in the foreshock [Schwartz et al., 2000].

[8] The two hot flow anomalies in the Venus foreshock, labeled HFA \#1 and \#2 in Figure 3a, are shown in more detail in Figure 3b. The central low-magnetic field intensity regions in the HFAs are believed to be formed by inwarddirected electric fields set up by interplanetary current sheets passing through the foreshock [Schwartz et al., 2000]. Necessary conditions for the formation of HFAs are: (a) the motional electric field must be directed toward the current sheet on at least one side of the disturbance; and (b) the current sheet must move along the bow shock at a sufficiently slow speed so that particles reflected at the bow shock may propagate upward along the interplanetary current sheet [Schwartz et al., 2000]. Indeed, examination of the MESSENGER data shows clearly that these two HFAs are centered on weak interplanetary current sheets indicated by the change in the magnetic field before and after each HFA. Analysis of the magnetic field data around these cavities, following the approach of Schwartz et al. [2000], confirms that these requirements are satisfied for the
MESSENGER HFAs. The normals to the interplanetary current sheets, determined by taking cross products of the magnetic field before and after the HFA encounters, are found to be tilted by $\sim 60-67^{\circ}$ from the Sun-Venus line with an estimated error of $\sim 10^{\circ}$. The motional electric field points toward the current sheet on either side of the second HFA, but only on the pre-sector in the first event, explaining why the field at the edges of the second HFA is much stronger. Moreover, the ratio of the speed at which the current sheet "slides" along the bow shock to the gyro speed of reflected particles, again following Schwartz et al. [2000], is found to be 0.5 and 0.4 for the two events, respectively. Values less than unity imply that the reflected particles have time to move along the current sheet and to develop an extended cavity. Hence, there is little doubt that MESSENGER observed Earth-like HFAs during its passage through the Venus foreshock.

\section{Two-Spacecraft Determination of the Timescale for Draped Magnetic Flux Transport}

[9] A full understanding of the solar wind interaction with unmagnetized bodies such as comets and Venus will require knowledge of the three-dimensional flow field [Benna et al., 2009]. Two-point observations of time delay between the orientation of the magnetic field in the interplanetary medium and the magnetic field convected by the solar wind into the interaction region is a powerful test of global numerical models that has been used previously at comets [Schwingenschuh et al., 1987]. The MESSENGER Venus Express encounter geometry and the highly variable IMF clock angle during this interval are ideal for the determination of such flow-field time delays. As shown in 

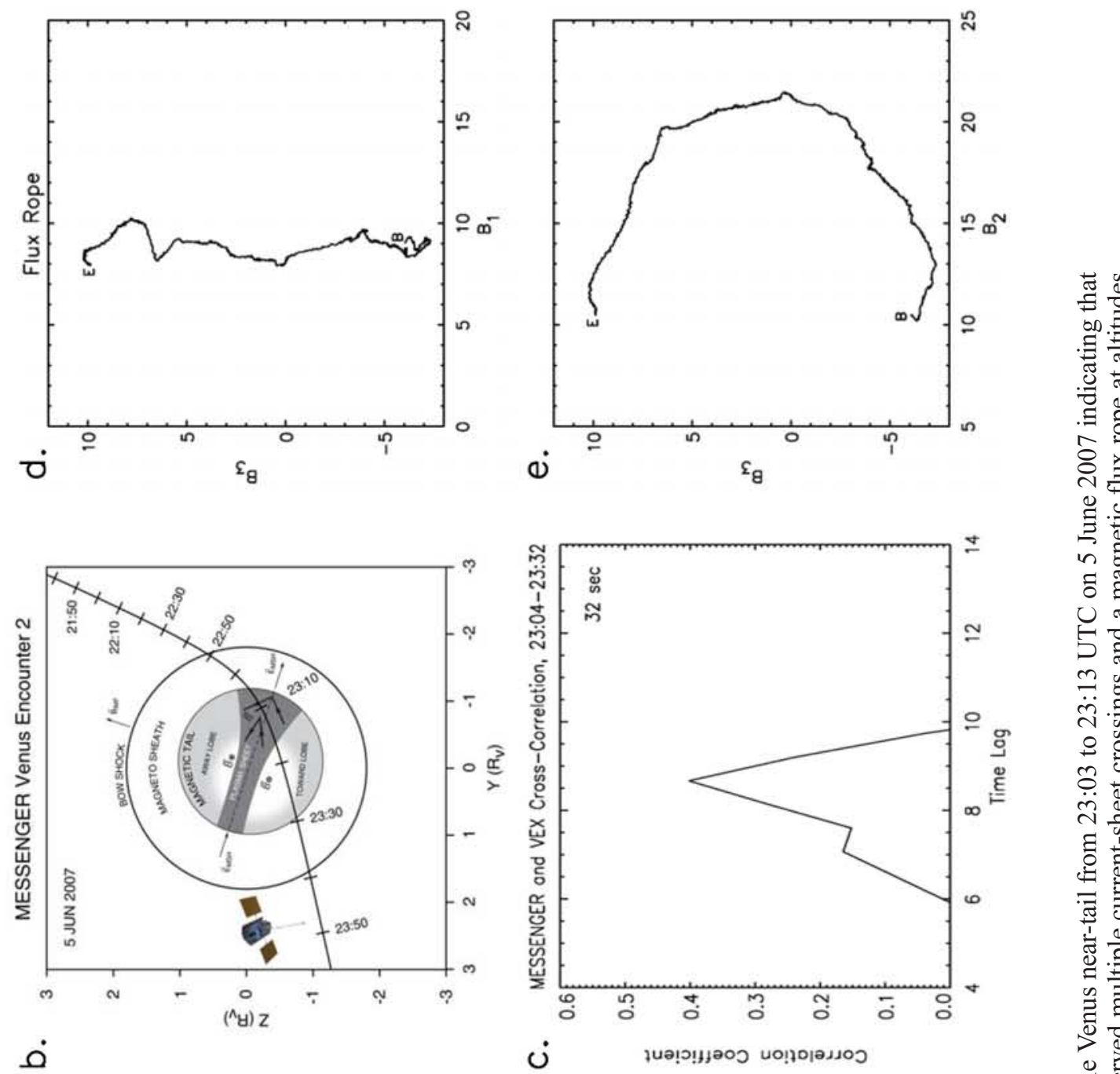

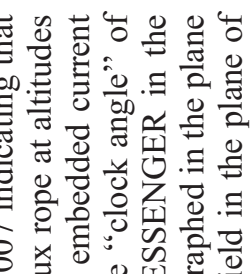

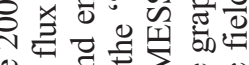

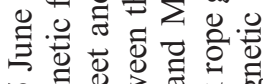

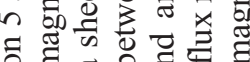

记

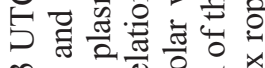

$\rightarrow$ co

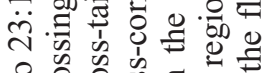

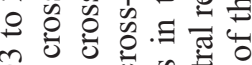

ஸे.

तิ

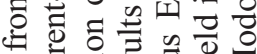

可

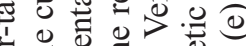

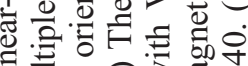

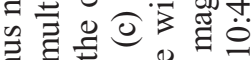

एँ

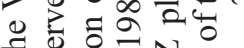

ف

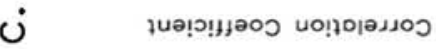

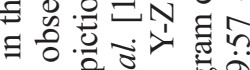

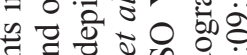

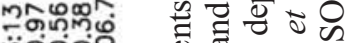

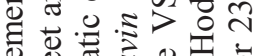

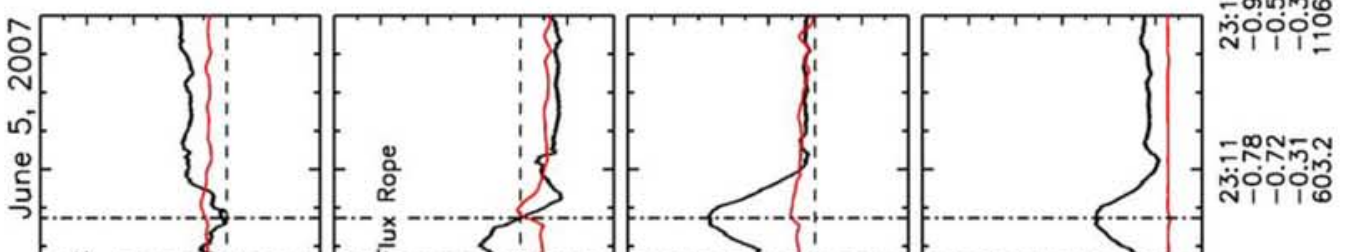

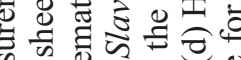

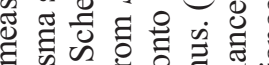

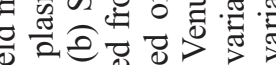
O

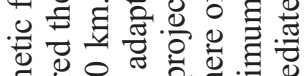
50

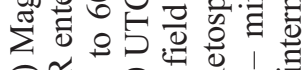
ङ

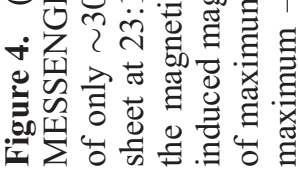

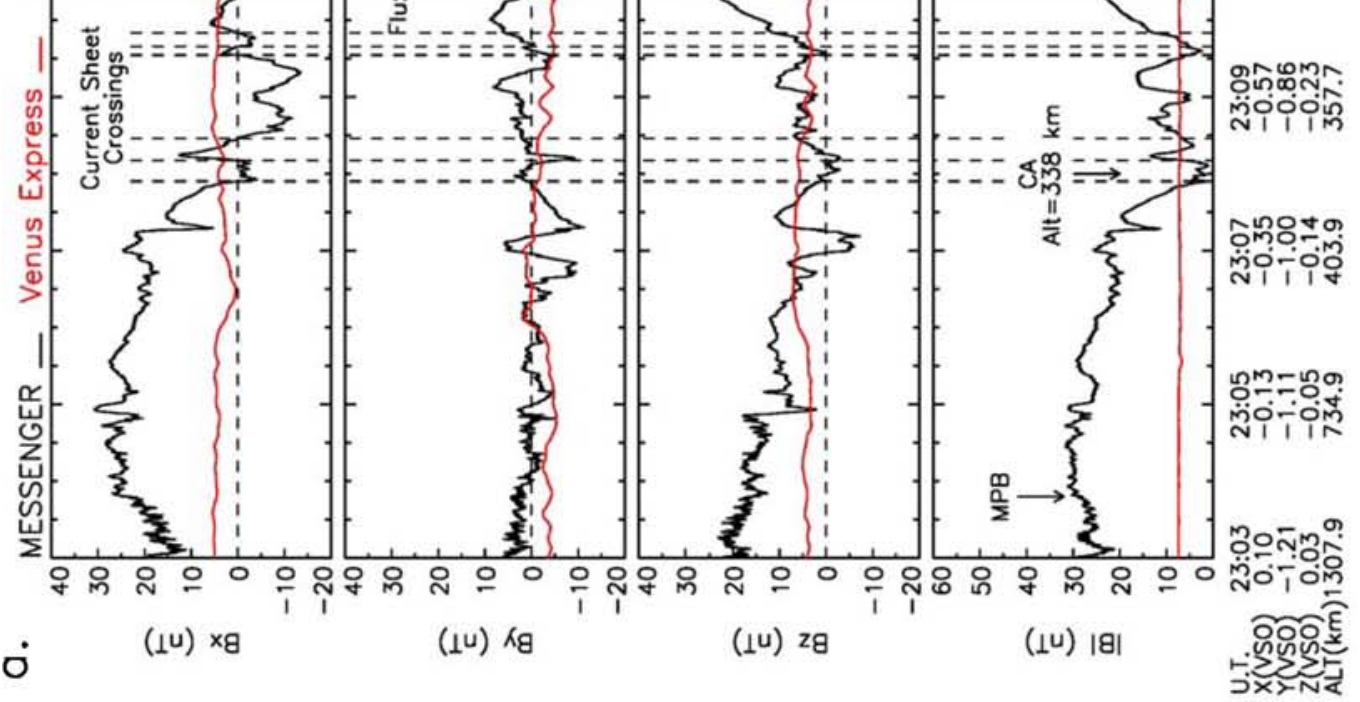


Figure 1, VEX was located essentially south of Venus and measured the IMF orientation nearly simultaneously with its arrival at the nose of the interaction region. Hence, to first order, there is no need to include time corrections for the transport between VEX and Venus. The duration of MESSENGER's immersion in the draped-field magnetosphere of Venus from the dayside MPB to the exit from the tail was $\sim 28 \mathrm{~min}$. A lagged cross-correlation between 32-s averages of IMF clock angle measured at VEX and MESSENGER (Figure 4c) shows a strong peak at $8.5 \mathrm{~min}$, indicating that this is the average amount of time it took for a change in IMF orientation applied to the front of the Venus magnetosphere to appear in the draped magnetic field between $\mathrm{X} \sim 0$ and $-3 \mathrm{R}_{\mathrm{V}}$. Presumably the effect of the IMF direction shift would be seen with smaller delays closer to the planet, but with the data from just this single flyby it was not possible to determine reliable time lags from smaller subsets of the encounter data set. The results obtained here are nonetheless in good agreement with similar determinations from Venera 9 and 10 [Yeroshenko, 1979] and Galileo - Pioneer Venus Orbiter [Huddleston et al., 1996] as well as from magnetohydrodynamic models [Benna et al., 2009].

\section{Near-Tail Magnetic Flux Rope}

[10] The magnetic field measurements in Figure 4a display the signature of a well-defined magnetic flux rope centered at 23:10:18, the first report of one observed in the Venus plasma sheet. Figures $4 \mathrm{~d}$ and $4 \mathrm{e}$ display hodograms of the magnetic field variation in the central portion surrounding the peak intensity. The directions of minimum, $\mathrm{B}_{1}=$ $(0.94,-0.05,0.34)$, intermediate, $B_{2}=(-0.33,0.15,0.93)$, and maximum, $\mathrm{B}_{3}=(-0.10,-0.99,0.12)$, variance are reasonably well defined; the ratios of maximum to intermediate and intermediate to minimum eigenvalue are 37.5 and 3.1 , respectively. The non-zero mean $\mathrm{B}_{1}=8.9 \mathrm{nT}$ indicates that the MESSENGER trajectory through the flux rope was off-center. The unipolar $\mathrm{B}_{2}$ and bipolar $\mathrm{B}_{3}$ traces are well behaved and correspond to the axial and transverse components of the flux rope field [Elphic et al., 1980; Vignes et al., 2004]. The duration of the flux rope, $\sim 30 \mathrm{~s}$, is too brief to be resolved by the MESSENGER Fast Imaging Plasma Spectrometer (FIPS). However, FIPS measurements in the surrounding plasma sheet (not shown) indicate a flow speed of $\sim 60 \mathrm{~km} / \mathrm{s}$. The estimated diameter of the flux rope is therefore $\sim 1800 \mathrm{~km}$, or $\sim 0.3 \mathrm{R}_{\mathrm{V}}$.

[11] Flux ropes with magnetic field signatures similar to those observed by MESSENGER are frequently observed in the ionospheres of Venus [Elphic et al., 1980] and Mars [Vignes et al., 2004]. However, these ionospheric flux ropes are much smaller and have diameters of order $10^{1}-10^{2} \mathrm{~km}$. They are believed to form in velocity shear layers in the inner magnetosheath or as a result of a Kelvin-Helmholtz instability at the ionopause [Wolff et al., 1980]. Thus far, searches for the signatures of reconnection in the cross-tail current layer at Venus, such as flux ropes, have yielded only negative results [Saunders and Russell, 1986; Slavin et al., 1989].

[12] These two possible mechanisms for the formation of the flux rope seen by MESSENGER differ greatly in the flux rope orientation relative to the cross-tail current sheet.
If the flux rope observed by MESSENGER were produced as a result of interplanetary flux tubes being "rolled up" near the ionopause as they slip about Venus, then the orientation of the flux rope should be approximately normal to the cross-tail current layer. Alternatively, if the flux rope seen by MESSENGER was caused by reconnection in the cross-tail current sheet, then its central axis should lie in the plane of the current sheet. The minimum variance analysis in Figure 4 shows that the orientation of the core magnetic field at the center of the flux rope is largely in the $\mathrm{Z}$ direction or perpendicular to the cross-tail current sheet. Hence, the MESSENGER magnetic field measurements suggest that it was formed not by reconnection, but rather by the velocity shear or some large-scale instability such as Kelvin-Helmholtz operating near the interface between the ionopause and inner magnetosheath [Wolff et al., 1980; Terada et al., 2004].

\section{Summary}

[13] The simultaneous Venus Express and MESSENGER measurements taken on 5-6 June 2007 provide a rare chance to observe the influence of solar wind plasma and interplanetary magnetic field conditions on the solar wind interaction with Venus. The MESSENGER and VEX magnetic field measurements clearly show the presence of welldeveloped HFAs in the Venus foreshock, the first time such structures have been reported at Venus. The importance of these HFAs at Venus is that they form under a very different set of solar wind conditions from those seen at other planets [Øieroset et al., 2001; Masters et al., 2008]. Moreover, these HFAs may significantly perturb conditions in the solar wind and influence the downstream interaction with the ionosphere and exosphere.

[14] The MESSENGER and VEX magnetic field measurements have been used to determine the timescale for a change in IMF orientation to be transported into the induced magnetosphere between $\mathrm{X} \sim 0$ and $-3 \mathrm{R}_{\mathrm{V}}$. The result, $8.5 \mathrm{~min}$, is similar to those found earlier using Venera 9 and 10 [Yeroshenko, 1979] and Galileo - Pioneer Venus Orbiter [Huddleston et al., 1996] observations. It also supports the results of recent fluid modeling of the Venus-solar wind interaction [Benna et al., 2009].

[15] The MESSENGER magnetic field measurements also captured a well-defined magnetic flux rope embedded within the Venus plasma sheet. The orientation of the flux rope strongly suggests that it is produced by velocity shear or a large-scale Kelvin-Helmholtz instability [Wolff et al., 1980; Terada et al., 2004] operating near the interface between the ionopause and inner magnetosheath.

[16] Acknowledgments. Computational assistance and data visualization support provided by C. Liebrecht are gratefully acknowledged. The MESSENGER project is supported by the NASA Discovery Program under contracts NASW-00002 to the Carnegie Institution of Washington and NAS5-97271 to the Johns Hopkins University Applied Physics Laboratory.

\section{References}

Barabash, S., et al. (2007), The loss of ions from Venus through the plasma wake, Nature, 450, 650-653.

Benna, M., et al. (2009), Modeling the response of the induced magnetosphere of Venus to changing IMF direction using MESSENGER and Venus Express observations, Geophys. Res. Lett., 36, L04109, doi:10.1029/2008GL036718. 
Bertucci, C., et al. (2003), Magnetic field draping enhancement at the Martian magnetic pileup boundary from Mars Global Surveyor observations, Geophys. Res. Lett., 30(2), 1099, doi:10.1029/2002GL015713.

Elphic, R. C., C. T. Russell, J. A. Slavin, and L. H. Brace (1980), Observations of the dayside ionopause and ionosphere of Venus, J. Geophys. Res., 85, 7679-7696.

Huddleston, D. E., C. T. Russell, M. G. Kivelson, and J. G. Luhmann (1996), Time delays in the solar wind flow past Venus: Galileo-Pioneer Venus correlations, J. Geophys. Res., 101, 4456-4539.

Martinecz, C., et al. (2008), Location of the bow shock and ion composition boundaries at Venus-Initial determinations from Venus Express ASPERA-4, Planet. Space Sci., 56, 780-784.

Masters, A., C. S. Arridge, M. K. Dougherty, C. Bertucci, L. Billingham, S. J. Schwartz, C. M. Jackman, Z. Bebesi, A. J. Coates, and M. F. Thomsen (2008), Cassini encounters with hot flow anomaly-like phenomena at Saturn's bow shock, Geophys. Res. Lett., 35, L02202, doi:10.1029/2007GL032371.

McKenna-Lawlor, S. M. P., et al. (2008), Predicting interplanetary shock arrivals at Earth, Mars, and Venus: A real-time modeling experiment following the solar flares of 5-14 December 2006, J. Geophys. Res., 113, A06101, doi:10.1029/2007JA012577.

Øieroset, M., D. L. Mitchell, T. D. Phan, R. P. Lin, and M. H. Acuña (2001), Hot diamagnetic cavities upstream of the Martian bow shock, Geophys. Res. Lett., 28, 887-890.

Pérez-de-Tejada, H. (2004), Plasma channels and electron density profiles near the midnight plane in the Venus nightside ionosphere, J. Geophys. Res., 109, A04106, doi:10.1029/2002JA009811.

Saunders, M. A., and C. T. Russell (1986), Average dimensions and magnetic structure of the distant Venus magnetotail, J. Geophys. Res., 91, 5589-5604.

Schwartz, S. J., G. Paschmann, N. Sckopke, T. M. Bauer, M. Dunlop, A. N. Fazakerley, and M. F. Thomsen (2000), Conditions for the formation of hot flow anomalies at Earth's bow shock, J. Geophys. Res., 105, 12,63912,650 .

Schwingenschuh, K., W. Riedler, Y. Yeroshenko, J. L. Phillips, C. T. Russell, J. G. Luhmann, and J. A. Fedder (1987), Magnetic field draping in the comet Halley coma: Comparison of VEGA observations with computer simulations, Geophys. Res. Lett., 14, 640-643.

Slavin, J. A., R. E. Holzer, J. R. Spreiter, and S. S. Stahara (1984), Planetary Mach cones: Theory and observation, J. Geophys. Res., 89, $2708-$ 2714.
Slavin, J. A., D. S. Intriligator, and E. J. Smith (1989), Pioneer Venus Orbiter magnetic field and plasma observations within the Venus magnetotail, J. Geophys. Res., 94, 2383-2398.

Terada, N., H. Shinagawa, and S. Machida (2004), Global hybrid model of the solar wind interaction with the Venus ionosphere: Ion escape processes, Adv. Space Res., 33, 161-166.

Vignes, D., M. H. Acuña, J. E. P. Connerney, D. H. Crider, H. Rème, and C. Mazelle (2004), Magnetic flux ropes in the Martian atmosphere: Global characteristics, Space Sci. Rev., 111, 223-231.

Wolff, R. S., B. E. Goldstein, and C. M. Yeates (1980), The onset and development of Kelvin-Helmholtz instability at the Venus ionopause, J. Geophys. Res., 85, 7697-7707.

Yeroshenko, Y. G. (1979), Unipolar induction effects in the magnetic tail of Venus, Kosm. Issled., 17, 93-105.

Zhang, T. L., J. G. Luhmann, and C. T. Russell (1991), The magnetic barrier at Venus, J. Geophys. Res., 96, 11,145-11,153.

Zhang, T. L., et al. (2008), Initial Venus Express magnetic field observations of the Venus bow shock location at solar minimum, Planet. Space Sci., 56, 785-789.

B. J. Anderson, R. E. Gold, G. C. Ho, H. Korth, S. M. Krimigis, and R. McNutt Jr., Johns Hopkins University Applied Physics Laboratory, 11100 Johns Hopkins Road, Laurel, MD 20723, USA.

S. Barabash, Swedish Institute of Space Physics, Box 812, SE-981 28 Kiruna, Sweden.

M. Benna, Solar System Exploration Division, NASA Goddard Space Flight Center, 8800 Greenbelt Road, Greenbelt, MD 20771, USA.

S. A. Boardsen, M. Sarantos, and J. A. Slavin, Heliophysics Science Division, NASA Goddard Space Flight Center, Code 670, Greenbelt, MD 20771, USA. (james.a.slavin@nasa.gov)

M. Fraenz, Max Planck Institute for Solar System Research, Max-PlanckStrasse 2, D-37191 Katlenburg-Lindau, Germany.

G. Gloeckler, J. M. Raines, and T. H. Zurbuchen, Department of Atmospheric, Oceanic and Space Sciences, University of Michigan, 2455 Hayward Street, Ann Arbor, MI 48109, USA.

S. C. Solomon, Department of Terrestrial Magnetism, Carnegie Institution of Washington, 5241 Broad Branch Road, NW, Washington, DC 20015, USA.

T. Zhang, Space Research Institute, Austrian Academy of Sciences, Schmiedlstrasse 6, A-8042 Graz, Austria. 